\title{
Application of Fuzzy Linear Programming to Transportation Planning Decision Problems with Multiple Fuzzy Goals
}

\author{
Tien-Fu Liang ${ }^{1}$ \\ ${ }^{1}$ Department of Industrial Management, Hsiuping Institute of Technology, 11 Gungye Road, \\ Dali City, Taichung, Taiwan 412, R.O.C. \\ E-mail: farmer@mail.hit.edu.tw
}

\begin{abstract}
This work develops a fuzzy linear programming (FLP) method for solving the transportation planning decision (TPD) problems with fuzzy goals, available supply and forecast demand. The proposed method attempts to minimize the total production and transportation costs and the total delivery time with reference to available supply and machine capacities at each source, as well as forecast demand and warehouse space constraints at each destination. An industrial case is used to demonstrate the feasibility of applying the FLP method to a real TPD problem.
\end{abstract}

Keywords: Transportation planning decisions; Fuzzy linear programming; Fuzzy set theory

\section{Introduction}

In real-world transportation planning decision (TPD) problems, input data and related parameters, such as available supply and forecast demand, are often imprecise/fuzzy because some information is incomplete or unobtainable. Also, the decision maker (DM) must simultaneously handle conflicting goals that govern the use of the constrained resources within organizations. Particularly, these conflicting goals are required to be optimized simultaneously by the DM, frequently in the framework of fuzzy aspiration levels (Li and Lai, 2000; Abd El-Wahed, 2006). Obviously, conventional LP method and existing solution algorithms cannot solve all TPD programming problems in uncertain environments.

Zimmermann (1976) first introduced fuzzy set theory into the ordinary LP and the multi-objective linear programming (MOLP) problems with fuzzy goals and constraints. Subsequently, Zimmermann's fuzzy linear programming (FLP) has developed into several fuzzy optimization methods to solve TPD problems. Chanas et al. (1984) presented an FLP model for solving the TPD problems with crisp cost coefficients and fuzzy supply and demand. Moreover, Chanas and Kuchta (1996) proposed the concept of the optimal solution of the TPD problem with fuzzy coefficients expressed as L-R fuzzy numbers, and developed an algorithm for determining the solution. Furthermore, Li and Lai (2000) designed a fuzzy compromise programming method to obtain a non-dominated compromise solution for fuzzy TPD problems with multiple objectives in which various

objectives were synthetically considered with the marginal evaluation for individual objectives and the global evaluation for all objectives. More recently, Abd El-Wahed (2006) designed a fuzzy programming approach to determine the optimal compromise solution of a multi-objective TPD problem by measuring the degree of closeness of the compromise solution to the ideal solution using a family of distance functions.

This work develops an interactive FLP method for solving multi-objective TPD problems with fuzzy goals, available supply and forecast demand. The proposed method attempts simultaneously to minimize the total production and transportation costs and the total delivery

\section{Problem formulation}

\subsection{Problem description and notation}

Assume that a logistics center seeks to determine the transportation plan of a homogeneous commodity from $m$ sources to $n$ destinations. Each source has an available supply of the commodity to distribute to various destinations, and each destination has a forecast demand of the commodity to be received from various sources. This work focuses on developing an FLP method for optimizing the transportation plan in fuzzy environments.

The following notation is used.

- Index sets

$$
\begin{array}{ll}
i & \text { index for source, for all } i=1,2, \ldots, m \\
j & \text { index for destination, for all } j=1,2, \ldots, n \\
g & \text { index for objectives, for all } g=1,2, \ldots, k
\end{array}
$$

- Decision variables

$Q_{i j} \quad$ units transported from source $i$ to destination $j$ (units)

- Objective functions

$z_{1} \quad$ total production and transportation costs (\$)

$z_{2} \quad$ total delivery time (hours)

- Parameters

$p_{i j} \quad$ production cost per unit delivered from source $i$ to destination $j$ (\$/unit) 
$c_{i j} \quad$ transportation cost per unit delivered from source $i$ to destination $j$ (\$/unit)

$t_{i j} \quad$ transportation time per unit delivered from source $i$ to destination $j$ (\$/unit)

$\widetilde{S}_{i} \quad$ total available supply for each source $\mathrm{i}$ (units)

$\widetilde{D}_{j} \quad$ total forecast demand of each destination $j$ (units)

$a_{i j} \quad$ hours of machine usage per unit produced by each source $i$ (machine-hour/unit)

$M_{i \max }$ maximum machine capacities available for each source $i$ (machine-hour)

$B \quad$ total budget (\$)

$b_{i j} \quad$ warehouse space per unit delivered from source $i$ to destination $j$ ( $f t^{2} /$ unit)

$W_{i \max }$ maximum warehouse space available for each destination $j\left(f t^{2}\right)$

\subsection{Fuzzy multi-objective linear programming (FMOLP) model}

\subsubsection{Objective functions}

- Minimize total production and transportation costs

$$
\text { Min } z_{1} \cong \sum_{i=1}^{m} \sum_{j=1}^{n}\left(p_{i j}+c_{i j}\right) Q_{i j}
$$

- Minimize total delivery time

$$
\text { Min } z_{2} \cong \sum_{i=1}^{n} \sum_{j=1}^{m} t_{i j} Q_{i j}
$$

The symbol ' $\cong$ ' is the fuzzified version of ' $=$ ' and refers to the fuzzification of the aspiration levels.

\subsubsection{Constraints}

- Constraints on total available supply for each source $i$

$$
\sum_{j=1}^{n} Q_{i j} \leq \widetilde{S}_{i} \quad \forall i
$$

- Constraints on total forecast demand for each destination $j$

$$
\sum_{i=1}^{m} Q_{i j} \geq \widetilde{D}_{j} \quad \forall j
$$

- Constraints on total budget

$$
\sum_{i=1}^{m} \sum_{j=1}^{n}\left(p_{i j}+c_{i j}\right) Q_{i j} \leq B
$$

- Constraints on machine capacities for each source $i$

$$
\sum_{j=1}^{n} a_{i j} Q_{i j} \leq M_{i \max } \quad \forall i
$$

- Constraints on warehouse space for each destination $j$

$$
\sum_{i=1}^{m} b_{i j} Q_{i j} \leq W_{j \max } \quad \forall j
$$

- Non-negativity constraints on decision variables

$$
Q_{i j} \geq 0 \quad \forall i, \forall j
$$

\section{Model development}

\subsection{Membership functions}

The FLP approach developed herein exhibits greater computational efficiency and flexibility of the fuzzy arithmetic operations by employing the linear membership functions to represent fuzzy numbers for solving the multi-objective TPD problem in a fuzzy environment. The corresponding non-increasing continuous linear membership functions for all fuzzy objective functions can be formulated as follows.

$$
f_{g}\left(z_{g}\right)= \begin{cases}1 & z_{g} \leq z_{g}^{l} \\ \frac{z_{g}^{u}-z_{g}}{z_{g}^{u}-z_{g}^{l}} & z_{g}^{l}<z_{g}<z_{g}^{u} \quad g=1,2, \ldots, k \\ 0 & z_{g} \geq z_{g}^{u}\end{cases}
$$

where $z_{g}^{l}$ and $z_{g}^{u}, g=1,2, \ldots, k$, are the lower and upper bounds, respectively, of the $g$ th objective function $z_{g}$.

Moreover, the corresponding non-increasing continuous linear membership functions for the fuzzy constraint (3) can be defined as follows.

$$
f_{i}\left(H_{i}\right)= \begin{cases}1 & H_{i} \leq S_{i}^{l} \\ \frac{S_{i}^{u}-H_{i}}{S_{i}^{u}-S_{i}^{l}} & S_{i}^{l}<H_{i}<S_{i}^{u} \quad i=1,2, \ldots, m \\ 0 & H_{i} \geq S_{i}^{u}\end{cases}
$$

where $H_{i}=\sum_{j=1}^{n} Q_{i j}, i=1,2, \ldots, m ; S_{i}^{l}$ and $S_{i}^{u}$, $i=1,2, \ldots, m$, are the lower and upper bounds of the fuzzy resources, respectively, of the $i$ th fuzzy inequality constraint.

Similarly, the corresponding non-decreasing continuous linear membership functions for the fuzzy constraint (4) can be defined as follows. 


$$
f_{j}\left(V_{j}\right)= \begin{cases}1 & V_{j} \geq D_{j}^{u} \\ \frac{V_{j}-D_{j}^{l}}{D_{j}^{u}-D_{j}^{l}} & D_{j}^{l}<V_{j}<D_{j}^{u} \quad j=1,2, \ldots, n \\ 0 & V_{j} \leq D_{j}^{l}\end{cases}
$$

where $V_{j}=\sum_{i=1}^{m} Q_{i j}, j=1,2, \ldots, n ; D_{j}^{u}$ and $D_{j}^{l}$, $j=1,2, \ldots, n$, are the upper and lower bounds of the fuzzy resources, respectively, of the $j$ th fuzzy inequality constraint. The pattern of the nonincreasing continuous linear membership functions $f_{j}\left(V_{j}\right)$ is similar to $f_{g}\left(z_{g}\right)$.

\subsection{Solving the fuzzy MOLP problem}

The minimum operator is used to aggregate all fuzzy sets. Introducing the auxiliary variable $L$ enables the original FMOLP problem to be converted into an equivalent ordinary LP form. Consequently, the complete equivalent LP model for solving the fuzzy TPD problem can be formulated as follows.

$$
\begin{array}{lll}
\operatorname{Max} L & \\
\text { s.t. } & L \leq f_{g}\left(z_{g}\right) & \forall g \\
& L \leq f_{i}\left(H_{i}\right) & \forall i \\
& L \leq f_{j}\left(V_{j}\right) & \forall j
\end{array}
$$$$
\text { Eqs. (5) to (8) }
$$

\subsection{Solution procedure}

Step 1. Formulate the original FMOLP model for the TPD problems according to Eqs. (1) to (8).

Step 2. Specify the corresponding linear membership functions for all of the fuzzy objective functions and the fuzzy inequality constraints using Eqs. (9) and (11).

Step 3. Introduce the auxiliary variable $L$, and then transform the original FMOLP problem into an equivalent ordinary LP form using the minimum operator to aggregate all fuzzy sets.

Step 4. Solve the ordinary LP problem and obtain the initial compromise solution. If the DM is dissatisfied with the initial solution, the model should be modified until a satisfactory solution is obtained.

\section{Implementation}

\subsection{Data description}

Dali Company was used as a case study demonstrating the practicality of the proposed methodology. Table 1 lists the basic transportation data for the Dali case for the coming season. Related production data for all factories are listed in Table 2. The total budget is $\$ 300,000$. The maximum warehouse space for four distribution centers is as follows: 4,000 $f t^{2}$ (Taichung), 1,700 $f t^{2}$ (Haulien), $5,000 f t^{2}$ (Kaohsiung), and 5,800 $f t^{2}$ (Taipei).

\subsection{Solution procedure for the Dali case}

The corresponding non-increasing continuous linear membership functions for the objective functions can be defined using Eq. (9), as below.

$$
\begin{aligned}
& f_{1}\left(z_{1}\right)= \begin{cases}1 & z_{1} \leq 240,000 \\
\frac{800,000-z_{1}}{560,000} & 240,000<z_{1}<800,000 \\
0 & z_{1} \geq 800,000\end{cases} \\
& f_{2}\left(z_{2}\right)= \begin{cases}1 & z_{2} \leq 750,000 \\
\frac{2,250,000-z_{2}}{1,500,000} & 750,000<z_{2}<2,250,000 \\
0 & z_{2} \geq 2,250,000\end{cases}
\end{aligned}
$$

LINDO computer software is used to run the equivalent ordinary LP model. The results are $z_{1}=\$ 264,334, z_{2}=847,771$ hours, and the overall DM satisfaction is 0.9255 . Table 3 lists the optimal

\begin{tabular}{|c|c|c|c|c|c|}
\hline \multirow[b]{2}{*}{ Source } & \multicolumn{4}{|l|}{ Destination } & \multirow{2}{*}{$\begin{array}{l}\text { Available supply } \\
\text { (dozen bottles) }\end{array}$} \\
\hline & 1. Taichung & 2. Haulien & 3. Kaohsiung & 4. Taipei & \\
\hline 1. Changhua & $0.8^{*} / 6^{* *}$ & $3.0 / 40$ & $2.1 / 12$ & $1.8 / 16$ & {$[18,000,26,000]$} \\
\hline 2. Touliu & $1.3 / 10$ & $3.6 / 32$ & $1.6 / 15$ & $2.5 / 22$ & {$[24,000,32,000]$} \\
\hline 3. Hsinchu & $1.8 / 12$ & $3.5 / 30$ & $2.4 / 18$ & $1.0 / 10$ & {$[13,000,18,000]$} \\
\hline $\begin{array}{l}\text { Forecast demand } \\
\text { (dozen bottles) }\end{array}$ & {$[8,000,12,500]$} & {$[3,000,6,500]$} & {$[9,500,16,500]$} & {$[12,000,21,000]$} & \\
\hline
\end{tabular}
TPD plan for the Dali case.

\section{Computational analysis}

Several significant finding regarding the practical application of the proposed FLP method are as follows. First, the interactive FLP method developed

Table 1. Summarized transportation data in the Dali case

Note: ${ }^{* *}$ denotes transportation cost per unit (in U.S. dollar); ${ }^{* * *}$ denotes delivery time per unit (hours). 
Table 2. Summarized production data in the Dali case

\begin{tabular}{lllll}
\hline & $\begin{array}{l}p_{i j} \\
\text { Factory }\end{array}$ & $\begin{array}{l}a_{i j} \\
\text { (machine-hour/unit) }\end{array}$ & $\begin{array}{l}M_{i \max } \\
\text { (machine-hours) }\end{array}$ & $b_{i j}\left(f t^{2} /\right.$ unit $)$ \\
\hline Changhua & 3.0 & 0.21 & 3800 & 0.32 \\
Touliu & 2.7 & 0.16 & 3900 & 0.28 \\
Hsinchu & 3.6 & 0.12 & 1600 & 0.30 \\
\hline
\end{tabular}


Table 3. Optimal transportation plan for the Dali case

\begin{tabular}{ll}
\hline Item & Solutions \\
\hline$Q_{i j}$ (in dozen bottles) & $Q_{11}=12,165, Q_{12}=0, Q_{13}=4,580, Q_{14}=0, Q_{21}=0, Q_{22}=0, Q_{23}=11,399$, \\
& $Q_{24}=13,197, Q_{31}=0, Q_{32}=6,239, Q_{33}=0, Q_{34}=7,133$. \\
$L$ value & $L=0.9255$ \\
Objective values & $z_{1}=\$ 264,334 \quad z_{2}=847,771$ hours \\
\hline
\end{tabular}

Table 4. Comparisons of three main TPD methods

\begin{tabular}{llll}
\hline Factor & Chanas and Kuchta (1996) & Li and Lai (2000) & The proposed FLP method \\
\hline Objective function & Single/Fuzzy & Multiple/Fuzzy & Multiple/Fuzzy \\
Constraints property & Crisp & Crisp & Fuzzy \\
Membership function & L-R (Trapezoid) & Linear & Linear \\
Budget & Not considered & Not considered & Considered \\
Machine capacity & Not considered & Not considered & Considered \\
Warehouse space & Not considered & Not considered & Considered \\
Computational efficiency & Medium & Low & High \\
\hline
\end{tabular}

in this work yields an efficient compromise solution and presents the overall DM satisfaction levels with the determined goal values in a multi-objective TPD problem. For instance, the overall DM satisfaction with the determined goal values, $z_{1}=\$ 264,334$ and $z_{2}=847,771$ hours, in the Dali case initially was generated as 0.9255 . Moreover, if the DM did not accept the initial overall degree of this satisfaction value, then the $L$ value was adjusted by changing the fuzzy data and parameters to seek a better compromise solution.

Moreover, the DM generally faces a planning problem with multiple fuzzy objectives, when making a transportation decision. The proposed FLP method can satisfy the requirement for the practical application for solving TPD problems since it attempts simultaneously to minimize the total production and transportation costs and the total delivery time in a fuzzy environment.

Finally, the proposed FLP approach exhibits greater computational efficiency by employing the linear membership functions to represent fuzzy numbers. The main advantages of the linear membership function are the simplicity and flexibility of the fuzzy arithmetic operations (Zimmermann, 1978, 1996). Table 4 compares the FLP method proposed in this work to the Chanas and Kuchta (1996) and Li and Lai (2000) methods.

\section{Conclusions}

This work develops an interactive FLP method for solving the TPD problems with fuzzy goals, available supply and forecast demand. The proposed FLP method attempts simultaneously to minimize the total production and transportation costs and total delivery time. An industrial case is used to demonstrate the feasibility of applying the proposed FLP method to real-world TPD problems. Consequently, the interactive method developed in this work yields an efficient compromise solution and overall DM satisfaction with the given goal values.

\section{References}

[1] W. F. Abd El-Wahed, "Interactive fuzzy goal programming for multi-objective transportation problems," Omega, 34, pp. 158-166, 2006.

[2] R. E. Bellman and L. A. Zadeh, "Decisionmaking in a fuzzy environment," Management Science, 17, pp. 141-164, 1970.

[3] S, Chanas, W. Kolodziejczyk, and A. Machaj, "A fuzzy approach to the transportation problem," Fuzzy Sets and Systems, 13, pp. 211-222, 1984.

[4] S. Chanas and D. Kuchta, "A concept of the optimal solution of the transportation problem with fuzzy cost coefficients," Fuzzy Sets and Systems, 82, pp. 299-305, 1996.

[5] D. Dubois and H. Prade, "Systems of linear fuzzy constraints," Fuzzy Sets and Systems, 3, pp. 37-48, 1980.

[6] L. Li and K. K. Lai, "A fuzzy approach to the multiobjective transportation problem," Computers and Operations Research, 27, pp. 43-57, 2000.

[7] H.-J. Zimmermann, "Description and optimization of fuzzy systems," International Journal of General Systems, 2, pp. 209-215, 1976.

[8] H.-J. Zimmermann, "Fuzzy programming and linear programming with several objective functions," Fuzzy Sets and Systems, 1, pp. 45-56, 1978.

[9] H.-J. Zimmermann, Fuzzy Set Theory and Its Application. Boston: Kluwer, 1996. 
